\title{
Una reflexión sobre la relevancia de las actitudes hacia el cáncer
}

\author{
Ximena Palacios-Espinosa', Jenny Fernanda Vargas-Jiménez², María Paula Calle-Nassiff²
}

\section{RESUMEN}

EI cáncer representa altas tasas de morbimortalidad humana, por ello su diagnóstico ha sido objeto de respuestas psicológicas diversas como las actitudes, las cuales denotan una tendencia de favorabilidad o desfavorabilidad hacia una entidad específica. Una revisión de la literatura publicada entre 2005 y 2017 sobre las actitudes hacia el cáncer, analizadas a través del modelo tripartito, dio lugar a reflexionar sobre la importancia que estas tienen, tanto para los pacientes, como para la población general y los profesionales de la salud. De acuerdo con lo anterior, se presentan las actitudes reportadas hacia el cáncer, la forma en que interactúan los conocimientos, las creencias, las emociones y las conductas y, también, las conclusiones de una postura crítica y analítica frente a estas actitudes con sus implicaciones para los profesionales de la salud.

\section{PALABRAS CLAVE}

Actitudes; Afecto; Cáncer; Conducta; Conocimiento; Emoción

\section{SUMMARY}

\section{A reflection on the relevance of attitudes towards cancer}

Cancer represents high rates of human morbidity and mortality and for this reason, its diagnosis has been object of diverse psychological responses, such as attitudes, which denote a tendency of favorability or unfavorability towards a specific entity. A review of the literature

\footnotetext{
1 Psicóloga, Universidad El Bosque. Bogotá, Colombia.

2 Psicóloga, Universidad del Rosario, Bogotá, Colombia.

Correspondencia: Jenny Fernanda Vargas-Jiménez; jenny.vargas@urosario.edu.co Recibido: diciembre 12 de 2017

Aceptado: junio 20 de 2018
}

Cómo citar: Palacios-Espinosa X, Vargas-Jiménez JF, Calle-Nassiff MP. Una reflexión sobre la relevancia de las actitudes hacia el cáncer. latreia. 2019 Abr-Jun; 32(2):126-132. D0I 10.17533/udea.iatreia.03. 
published between 2005 and 2017 on attitudes toward cancer, led to reflection on the importance they have for both patients and the general population and health professionals. In this way, presented in this reflection, the attitudes reported towards this disease, the way in which knowledge, beliefs, emotions and behaviors interact and the conclusions of a critical and analytical approach to these attitudes and their implications for professionals of the Health.

\section{KEY WORDS}

Affect; Attitudes; Behavior; Emotion; Knowledge; Neoplasms

\section{REFLEXIÓN}

EI emperador de todos Ios males, así titula, en parte, una de sus recientes obras Siddartha Mukherjee. En ella, relata con minucioso detalle la historia del cáncer, responsable de la muerte de millones de seres vivos en la historia de la tierra y de cuyos orígenes en la humanidad hay antiquísima evidencia (1).

El cáncer ha sido, y según los pronósticos seguirá siendo, una de las primeras causas de muerte en el mundo (2). Ciertamente, esta enfermedad ha "reinado" sin mayor competencia, como una de las enfermedades más mortales y por consiguiente, más temidas por la humanidad. Se ha librado una lucha incesante para detenerla y siglos después, no se ha logrado alcanzar esa meta (3). Las consecuencias de su curso y de los tratamientos médicos disponibles han mantenido la idea de que es sinónimo de muerte y sufrimiento (4).

Por consiguiente, es comprensible que el cáncer haya sido, entre otros, objeto de estigma (5), prejuicio (6), discriminación (7) y diversidad de actitudes (8).

Las actitudes, objeto de esta reflexión, son un concepto psicológico ampliamente investigado, entre otras, por su capacidad de actuar en todos los ámbitos de la influencia social (individual, interpersonal e intergrupal) (9). A mediados de la década de los años 30 del siglo XX, Allport definió las actitudes como un estado psicológico y neurológico que se organiza a partir de la experiencia y ejerce influencia dinámica en su respuesta ante objetos y situaciones con los cuales se relaciona (10). En el siglo XXI, otros autores han definido las actitudes como "una organización de creencias y cogniciones en general duraderas, dotadas de cargas afectivas a favor o en contra de un objeto social definido, que predispone a una acción coherente con las cogniciones y afectos relativos a dicho objeto" (11).

De manera general, se ha aceptado que las actitudes tienen un componente cognitivo (conocimientos $y$ creencias), emocional (reacciones emocionales y sentimientos) y comportamental (conducta e intenciones conductuales) (9). Específicamente, el modelo tripartito ha sido uno de los modelos teóricos para estudiar las actitudes. De acuerdo con este, "Ias actitudes consisten en elementos afectivos (sentimientos, emociones), cognoscitivos (creencias, asociaciones percibidas entre el objeto actitudinal $y$ sus atributos) y comportamentales (intenciones comportamentales o comportamientos efectivos)" (12). Además, incluye varios factores del individuo que facilitan, u obstaculizan, la presentación de determinada conducta (13).

Es pertinente resaltar que el modelo tripartito surge aproximadamente en 1940 y tuvo mayor auge en la década de los años sesenta del sioglo XX, por su utilización clínica en los tratamientos para modificar tanto conductas como actitudes y por su amplia inclusión en Ios libros de Psicología Social (14). No obstante, entre los investigadores este modelo fue criticado por centrarse en el afecto o emoción más que en los otros componentes de la actitud, y por considerar que los componentes podían sobreponerse (14). Si bien estos componentes son distinguibles, no siempre se correlacionan. Esto puede restar claridad a lo que se está midiendo específicamente cuando se estudian las actitudes, por lo tanto, los investigadores deben ser precavidos a la hora de establecer relaciones de causalidad (14). Pese a lo anterior, la relación entre las actitudes y la conducta ha sido ampliamente estudiada (15) y se ha concluido que las primeras son predictoras al explicar el comportamiento humano en un marco contextual específico (16-17). En consecuencia, las actitudes que las personas han desarrollado hacia el cáncer tienen un efecto en la forma como se comportan ante este diagnóstico.

Tan diversos son los objetos como las actitudes que se generan hacia ellos. Las enfermedades han sido, históricamente, objeto actitudinal. Así lo describen diversas publicaciones científicas (18-20) y de 
divulgación (1, 21); de manera magistral, Susan Sontag (2012), en su obra La enfermedad y sus metáforas, alude al impacto emocional que el cáncer y el Sida han provocado en la humanidad (21).

En consonancia con lo anterior, el objetivo de este escrito fue reflexionar en torno a las actitudes hacia el cáncer, estudiadas desde el modelo tripartito, a partir de una revisión exhaustiva de la literatura publicada entre 2005 y 2017.

\section{ACTITUDES HACIA EL CÁNCER}

En lo referente al componente cognitivo de las actitudes, la literatura indica que algunas creencias sobre el cáncer incluyen que es un castigo de Dios, Alá o una prueba de vida (22-26); una enfermedad contagiosa $(22,27,28)$; que se produce por estrés y estilos de vida no saludables (29,30), "mal comportamiento" (ej. homosexualidad, promiscuidad) (28,31-33); suerte o casualidad $(33,34)$; lesiones previas $(35,36)$ o infecciones $(25)$.

Las reacciones emocionales y sentimientos hacia el cáncer incluyen preocupación relacionada con desarrollar la enfermedad (26) o que personas cercanas la padezcan (37); miedo, fatalismo, pesimismo, preocupación, desesperanza, impotencia, perturbación emocional y estrés respecto al curso de la enfermedad $(22,23,26,34,35,37,38-44)$.

En relación con el comportamiento, la literatura disponible indica que el cáncer genera intenciones conductuales como la disposición a tomar decisiones sobre el estilo de vida que se ha tenido, dependiendo de la información sobre la prevención de la enfermedad de la que se dispone $(45,46)$ y de la creencia de que el estado de salud es controlado por sus conductas $(34,37)$

Los componentes descritos interactúan definiendo así el complejo actitudinal hacia el cáncer.

Donnelly et al. (2013), encontraron que las personas que creen que el cáncer es un castigo de Dios tienden a no tener conductas de prevención y detección temprana de la enfermedad (24). Quienes creen que la enfermedad es el resultado de un castigo divino por su mal comportamiento, sienten culpa de tenerla (32). Además, el miedo al cáncer se debe a creer que la enfermedad es mortal, intratable o incurable $(41,47,48)$.
Los conocimientos y las creencias, afectan las prácticas de prevención del cáncer de seno (49), como el autoexamen (50-52). Okobiaet al. (2006), encontraron en su estudio que las mujeres con mayores conocimientos sobre el cáncer de seno eran más propensas a aceptar la mastectomía como una forma de tratamiento, en comparación con las mujeres con menores conocimientos (25). Así mismo, encontraron que aunque la mamografía y el autoexamen son pruebas de detección temprana del cáncer, las participantes del estudio no llevan a cabo estas prácticas con frecuencia (25).

Por otro lado, en un estudio realizado en Turquía a mujeres mayores de 20 años, se encontró que si se aumenta el nivel de conocimiento sobre el cáncer de seno, se eleva la conciencia sobre la importancia del diagnóstico temprano y, en consecuencia, se aumenta la realización del autoexamen y la mamografía (53). De acuerdo con lo anterior, el conocimiento es un factor mediador para que se presente un cambio conductual (54) y por tanto, el conocimiento sobre el cáncer y las creencias culturales, influyen en el tipo de tratamiento que se busque $(22,23,25,33)$.

Las actitudes negativas hacia el cáncer afectan de forma también negativa a la comunicación del diagnóstico a otros (4) y a la realización del autoexamen de seno (18). Así, en algunos países como Francia e Italia, solo se comunica el diagnóstico a la familia por considerar que no es tolerable por parte de los pacientes (21). En contraste, una actitud positiva hacia la prevención conlleva al uso de métodos de detección temprana de la enfermedad, como el autoexamen (51). Según Sontag (2012), mientras se trate el cáncer como un ente desconocido e invencible y no como un padecimiento físico, la mayoría de los pacientes con este diagnóstico sentirán desesperanza al enterarse del mismo (21). En consecuencia, la solución para Sontag reside en desmitificar y rectificar las ideas que tiene el paciente sobre la enfermedad y no en ocultarle la verdad (21).

\section{CONCLUSIONES}

Las actitudes hacia el cáncer son predominantemente negativas. Algunos hallazogos, aún incipientes e insuficientes, indican que esto puede verse afectado por variables sociodemográficas (edad, género, nivel educativo, estado civil) y culturales (cultura de origen y religión). Estos dos datos son importantes, pues 
recuerdan la pertinencia de conocer el perfil de nuestros pacientes, de reconocerlo como factor predictor de sus actitudes y por ende, de sus posibles conductas ante la enfermedad (prevención primaria, de detección precoz, la búsqueda de ayuda profesional o la elección del tratamiento). El contexto sociocultural de cada paciente es, entre otros, el nicho en el que se aprenden y refuerzan conductas relacionadas con la enfermedad, se le da sionificado y se desarrollan actitudes hacia ella. De hecho, tan determinantes han sido las actitudes hacia el cáncer y en general el significado que este tiene para las personas, que tras haber sido paciente oncológico, se adquieren diversas identidades: paciente, persona que tuvo cáncer, víctima y sobreviviente (55).

Como pudo evidenciarse, las emociones frente al cáncer también son fundamentalmente negativas, con un predominio importante del componente de la ansiedad.

Esto permite inferir que la tendencia será a considerarla amenazante y por tanto, a afrontarla por medio de la evitación, la supresión emocional o la lucha $(56,57)$. En consecuencia, todo lo que represente al cáncer (profesionales de la salud, tratamiento, sistema, etc.) puede ser considerado como una amenaza y por lo tanto, pueden intentar protegerse asumiendo una postura defensiva.

De alguna manera, esto permitiría comprender que las conductas emitidas por los pacientes $y$ por los profesionales de la salud parezcan o resulten eventualmente aprehensivas para el otro. Sin embargo, siempre y sin excepciones, el paciente oncológico es el objetivo central del profesional de la salud $y$, de acuerdo con el planteamiento de Hopman y Rijken, se beneficiará del apoyo para aliviar su percepción de gravedad y amenaza del cáncer (57).

Contrario a lo que pueda considerarse, los profesionales de la salud, quienes día tras día se confrontan con la realidad del cáncer que amenaza la vida de sus pacientes, también tienen actitudes hacia este, y en consonancia con lo expuesto en esta reflexión, estas superan su conocimiento científico para mezclarse o afectarse por creencias y emociones propias. Por lo tanto, cabe preguntarse como profesional de la salud ¿cuál es su posición frente al cáncer?, ¿reconoce que sus actitudes afectan su comportamiento, la forma en que interactúa y se relaciona con sus pacientes?, ¿es consciente de que sus actitudes pueden llevarlo a emitir juicios de valor y debilitar su objetividad en el actuar profesional?, ¿cuántas veces se ha detenido a pensar como profesional de la salud en aquello que lo angustia en la cotidianidad de su trabajo, en la forma en que sus actitudes afectan a otros? y, ies funcional hacer caso omiso de sus emociones? Es probable que pensar sobre estos aspectos y reconocerlos contribuya de manera determinante en su proceder como profesional de la salud.

De acuerdo con lo anterior, es posible afirmar que las actitudes hacia una enfermedad se convierten en un elemento relevante que debe ser analizado, estudiado y considerado para facilitar la relación del profesional de la salud con el paciente.

Estudiar las actitudes desde el modelo tripartito puede resultar útil para definir cuáles son los componentes (cognitivo, emocional, conductual) predominantes en las actitudes hacia el cáncer y establecer así medidas de intervención oportunas, ideográficas y efectivas para modificarlas.

Variables psicológicas como las actitudes y cada uno de los componentes que las conforman, están presentes en todos los escenarios del trabajo que se desarroIla en los contextos de salud. Es un error considerarlas como de interés exclusivo de la ciencia psicológica. Al contrario, a partir de esta reflexión, es propositivo y pertinente, considerarlas como transversales en estos contextos y transdisciplinares. Por lo tanto, será de interés pensarlas como una variable central en la práctica y en la investigación de diversas ciencias de la salud, para promover así, el trabajo y la investigación interdisciplinarios.

\section{CONFLICTOS DE INTERESES}

Ninguno por declarar.

\section{REFERENCIAS BIBLIOGRÁFICAS}

1. Mukherjee S. El emperador de todos los males: Una biografía del cáncer. España: Taurus; 2014.

2. Organización Mundial de la Salud. Datos y cifras sobre el cáncer [Internet]. Ginebra: WHO; 2017 [citado Mayo 31 de 2017]. Disponible en: http://www.who.int/ cancer/about/facts/es/ 
3. Instituto Nacional del Cáncer [Internet]. Estados Unidos: National Institutes of Health; 2014. El pronóstico del cáncer. Disponible en: http://www.cancer.gov/espanol/cancer/diagnostico-estadificacion/ pronostico\#estad\%C3\%ADsticas-supervivencia

4. Cho J, Smith K, Choi EK, Kim IR, Chang YJ, Park HY, et al. Public attitudes toward cancer and cancer patients: a national survey in Korea. J Psychosoc Oncol. 2013 Mar;22(3):605-13. DOI 10.1002/pon.3041.

5. Palacios-Espinosa X, Zani B. La stigmatisation de la maladiephysique : le cas du cancer. J Psychosoc Oncol. 2012;6(4):189-200.

6. dos Santos Maia LF. Câncer de próstata: preconceitos, masculinidade e a qualidade de vida. Rev Latino-Am Enfermagem. 2012;2(6):16-20. DOI DOI: 10.24276/rrecien2177-157X.2012.2.6.16-20.

7. Gökler-Danışman I, Yalçınay-İnan M, Yiŏit İ. Experience of grief by patients with cancer in relation to perceptions of illness: The mediating roles of identity centrality, stigma-induced discrimination, and hopefulness. J Psychosoc Oncol. 2017 Nor-Dec;35(6):776-96. DOI 10.1080/07347332.2017.1340389.

8. Deniz S, Kurt B, Oğuzöncül AF, Nazlıcan E, Akbaba M, Nayir T. Knowledge, attitudes and behaviours of women regarding breast and cervical cancer in Malatya, Turkey. PLoS One. 2017 Nov;12(11):e0188571. DOI 10.1371/journal.pone.0188571.

9. Stainton Rogers W. Social Psychology. England: McGraw-Hill; 2011.

10. AllportGW. Attitudes. In: Murchison C, editor. Handbook of Social Psychology. Worchester, MA: Clark University; 1935. p. 798-844.

11. Rodrigues A. Psicología para principiantes: estudio de la interacción humana. México: Trillas; 2004.

12. García V, D’Adamo O. Actitudes y Conducta: Actitudes Políticas. En: Morales Domínguez JF, Paez D, Kornblit A, Asún D. Psicología Social. México: Prentice Hall; 2002. P. 287-307.

13. Reyes Rodríguez L. La teoría de la acción razonada: implicaciones para el estudio de las actitudes. INED. 2007;(7):66-77. Disponible en: file://C:/Users/Informatica/Downloads/Dialnet-LaTeoriaDeLaAccionRazonadaImplicacionesParaEIEstud-2358919.pdf
14. Breckler SJ. Empirical validation of affect, behavior, and cognition as distinct components of attitude. J PersSoc Psychol. 1984 Dec;47(6):1191-205.

15. Ajzen I. The theory of planned behaviour is alive and well, and not ready to retire: a commentary on Sniehotta, Presseau, and Araújo-Soares. HealthPsychol Rev. 2015;9(2):131-7. DOI 10.1080/17437199.2014.883474.

16. Kraus SJ. Attitudes and the prediction of behavior: a meta-analysis of the empirical literature. PersSocPsychol Bull; 1995,21(1):58-75. DOI 10.1177/0146167295211007.

17. Ajzen I, Fishbein M. Attitudes and the attitude-behavior Relation: Reasoned and automatic processes. En: Stroebe W, Hewstone M, editores. Europan review of social psychology [Internet]. Chichester, England: Wiley; 2000. p. 1-33. DOI 10.1080/14792779943000116.

18. Asuzu CC, Unegbu J, Akin-Odanye E. Knowledge, attitude and behaviour of the University of Ibadan women towards cancer of the cervix and its prevention. Psychooncology. 2012 Sep;21(9):1010-5. DOI 10.1002/ pon.2007.

19. Kujan O, Alzoghaibi I, Azzeghaiby S, Altamimi MA, Tarakji B, Hanouneh S, et al. Knowledge and attitudes of Saudi dental undergraduates on oral cancer. J Cancer Educ. 2014 Dec;29(4):735-8. DOI10.1007/ s13187-014-0647-5.

20. Sichanh C, Quet F, Chanthavilay P, Diendere J, Latthaphasavang $\nabla$, Longuet $C$, et al. Knowledge, awareness and attitudes about cervical cancer among women attending or not an HIV treatment center in Lao PDR. BMC Cancer. 2014 Mar;14:161. DOI 10.1186/1471-2407-14-161.

21. Sontag S. La enfermedad y sus metáforas: EI sida y sus metáforas [Internet]. España: Titivillus; 2012. Disponible en: http://ceiphistorica.com/wp-content/ uploads/2016/04/Susan-Sontag-La-enfermedad-y-susmet\%C3\%A1 foras.-El-sida-y-sus-met\%C3\%A1 foras.pdf

22. Ashing-Giwa KT, Padilla G, Tejero J, Kraemer J, Wright $\mathrm{K}$, Coscarelli A, et al. Understanding the breast cancer experience of women: a qualitative study of African American, Asian American, Latina and Caucasian cancer survivors. Psychooncology. 2004 Jun; 13(6):408-28.

23. Dein S. Explanatory models of and attitudes towards cancer in different cultures. Lancet Oncol. 2004 Feb;5(2): 119-24. 
24. Donnelly TT, AI Khater AH, AI-Bader SB, AI Kuwari MG, Al-Meer N, Malik M, et al. Beliefs and attitudes about breast cancer and screening practices among Arab women living in Qatar: a cross-sectional study. BMC Womens Health. 2013 Dec;13:49. DOI 10.1186/14726874-13-49.

25. Okobia MN, Bunker CH, Okonofua FE, Osime U. Knowledge, attitude and practice of Nigerian women towards breast cancer: a cross-sectional study. World J Surg Oncol. 2006 Feb 21;4:11.

26. Saleh M, Barlow-Stewart K, Meiser B, Tucker K, Eisenbruch M, Kirk J. Knowledge, attitudes and beliefs of Arabic-Australians concerning cancer. Psychooncology. 2012 Feb;21(2):195-202. DOI 10.1002/pon.1884.

27. Karbani G, Lim JN, Hewison J, Atkin K, Horgan K, Lansdown $M$, et al. Culture, attitude and knowledge about breast cancer and preventive measures: a qualitative study of South Asian breast cancer patients in the UK. Asian Pac J Cancer Prev. 2011;12(6):1619-26.

28. Aweke YH, Ayanto SY, Ersado TL. Knowledge, attitude and practice for cervical cancer prevention and control among women of childbearing age in Hossana Town, Hadiya zone, Southern Ethiopia: Community-based cross-sectional study. PLoS One. 2017 Jul;12(7):e0181415. DOI 10.1371/journal.pone.0181415.

29. Rabin C, Pinto B. Cancer-related beliefs and health behavior change among breast cancer survivors and their first-degree relatives. Psychooncology. 2006 Aug; 15(8):701-12.

30. Gorawara-Bhat R, O'Muircheartaigh S, Mohile S, Dale W. Patients' perceptions and attitudes on recurrent prostate cancer and hormone therapy: Qualitative comparison between decision-aid and control groups. J Geriatr Oncol. 2017 Sep;8(5):368-73. DOI 10.1016/j.jgo.2017.05.006.

31. Ali-Risasi C, Mulumba P, Verdonck K, VandenBroeck D, Praet $M$. Knowledge, attitude and practice about cancer of the uterine cervix among women living in Kinshasa, the Democratic Republic of Congo. BMC Womens Health. 2014 Feb;14(1):30. DOI 10.1186/14726874-14-30.

32. Muhbies FJ, Sheren AI-Doski NA. Attitude of patients toward cancer and its treatment. Int J Acad Res [Internet]. 2010;2(4):196-200. Available from: https:// www.researchgate.net/publication/311456203 ATTITUDE_OF_PATIENTS_TOWARD_CANCER_AND_ITS TREATMENTT
33. Die Trill M. Influencia de la cultura en la experiencia del cáncer. Psicooncología. 2003;(1):39-48.

34. Del Castillo A, Godoy-Izquierdo D, Vázquez ML, Godoy JF. Illness beliefs about cancer among healthy adults who have and have not lived with cancer patients. Int J Behav Med. 2011 Dec;18(4):342-51. DOI 10.1007/s12529-010-9141-6.

35. Dumalaon-Canaria JA, Hutchinson AD, Prichard I, Wilson $C$. What causes breast cancer? A systematic review of causal attributions among breast cancer survivors and how these compare to expert-endorsed risk factors. Cancer Causes Control. 2014 Jul;25(7):771-85. DOI 10.1007/s10552-014-0377-3.

36. Stein K, Zhao L, Crammer C, Gansler T. Prevalence and sociodemographic correlates of beliefs regarding cancer risks. Cancer. 2007 Sep;110(5):1139-48.

37. Kowalkowski MA, Hart SL, Du XL, Baraniuk S, Latini DM. Cancer perceptions: implications from the 2007 Health Information National Trends Survey. J Cancer Surviv. 2012 Sep;6(3):287-95. DOI 10.1007/s11764012-0217-y.

38. Dandash KF, AI-Mohaimeed A. Knowledge, attitudes, and practices surrounding breast cancer and screening in female teachers of buraidah, saudiarabia. Int J Health Sci (Qassim). 2007 Jan;1(1):61-71.

39. Geraghty JG, Zbar A, Costa A. Informing the public about advances in cancer therapy. Eur J Cancer Care (Engl). 2002 Mar;11(1):25-32.

40. Gilani SI, Khurram M, Mazhar T, Mir ST, Ali S, Tariq S, et al. Knowledge, attitude and practice of a Pakistani female cohort towards breast cancer. J Pak Med AsSOC. 2010 Mar;60(3):205-8.

41. Luquis RR, Villanueva Cruz IJ. Knowledge, attitudes, and perceptions about breast cancer and breast cancer screening among Hispanic women residing in South Central Pennsylvania. J Community Health. 2006 Feb;31(1):25-42.

42. McCaffery K, Wardle J, Waller J. Knowledge, attitudes, and behavioral intentions in relation to the early detection of colorectal cancer in the United Kingdom. Prev Med. 2003 May;36(5):525-35.

43. Odusanya OO, Tayo OO. Breast cancer knowledge, attitudes and practice among nurses in Lagos, Nigeria. Acta Oncol. 2001;40(7):844-8.

44. Quaife SL, Marlow LAV, McEwen A, Janes SM, Wardle J. Attitudes towards lung cancer screening in 
socioeconomically deprived and heavy smoking communities: informing screening communication. Health Expect. 2017 Aug;20(4):563-73. DOI 10.1111/hex.12481.

45. Keeney S, McKenna H, Fleming P, McIlfatrick S. Attitudes to cancer and cancer prevention: what do people aged 35-54 years think? Eur J Cancer Care (Engl). 2010 Nor;19(6):769-77. DOI 10.1111/j.1365-2354.2009.01137.x.

46. Duong DK, Shariff-Marco S, Cheng I, Naemi H, Moy LM, Haile R, et al. Patient and primary care provider attitudes and adherence towards lung cancer screening at an academic medical center. Prev Med Rep. 2017 Jan;6:17-22. DOI 10.1016/j.pmedr.2017.01.012.

47. Elmubarak E, Bromfield E, Bovell-Benjamin AC. Focused interviews with Sudanese Americans: perceptions about diet, nutrition, and cancer. Prev Med. 2005 May;40(5):502-9.

48. Jones DP. Knowledge, Beliefs and Feelings about Breast Cancer: The Perspective of African American Women. ABNF J. 2015 Winter;26(1):5-10.

49. Sudenga SL, Rositch AF, Otieno WA, Smith JS. Knowledge, attitudes, practices, and perceived risk of cervical cancer among Kenyan women: brief report. Int J GynecolCancer. 2013 Jun;23(5):895-9. DOI 10.1097/IGC.0b013e31828e425c.

50. Alharbi NA, Alshammari MS, Almutairi BM, Makboul G, EI-Shazly MK. Knowledge, awareness, and practices concerning breast cancer among Kuwaiti female school teachers. Alex J Med. marzo de 2012;48(1):7582. DOI 10.1016/j.ajme.2011.10.003.
51. Alwan NAS, AI-Diwan JKA, Al-Attar WM, Eliessa RA. Knowledge, attitude \&amp; practice towards breast cancer \&amp; breast self examination in Kirkuk University, Iraq. AsianPac J Reprod. 2012;1(4):308-11. DOI 10.1016/S2305-0500(13)60098-6.

52. Anaonostopoulos F, Spanea E. Assessing illness representations of breast cancer: a comparison of patients with healthy and benign controls. J Psychosom Res. 2005 Apr;58(4):327-34.

53. Ertem G, Dönmez YC, Dolgun E. Determination of the Health Belief and Attitude of Women Regarding Breast Cancer and Breast Self-Exam. J Breast Health. 2017 Apr;13(2):62-66. DOI 10.5152/tjbh.2017.3018.

54. Breslow RA, Sorkin JD, Frey CM, Kessler LG. Americans' knowledge of cancer risk and survival. Prev Med. 1997 Mar-Apr;26(2):170-7.

55. Park CL, Zlateva I, Blank TO. Self-identity after cancer: "survivor", "victim", "patient", and "person with cancer”. J Gen Intern Med. 2009 Nov;24 SuppI2:S430-5. DOI 10.1007/s1 1606-009-0993-X.

56. Cordova MJ, Giese-Davis J, Golant M, Kronnenwetter $C$, Chang $\nabla$, McFarlin S, et al. Mood disturbance in community cancer support groups. The role of emotional suppression and fighting spirit. J Psychosom Res. 2003 Nov;55(5):461-7.

57. Hopman P, Rijken M. Illness perceptions of cancer patients: relationships with illness characteristics and coping. Psychooncology. 2015 Jan;24(1):11-8. DOI 10.1002/pon.3591. 